$\mathbb{T}$ periodica polytechnica

Civil Engineering

$53 / 2$ (2009) 75,80

doi: $10.3311 /$ pp.ci.2009-2.03

web: http://www.pp.bme.hu/ci

(c) Periodica Polytechnica 2009

RESEARCH ARTICLE

\section{Test measurements by Eötvös torsion balance and gravimeters}

\author{
Géza Csapó / Sándor Laky / Csaba Égető / Zita Ultman / Gyula Tóth / Lajos Völgyesi
}

Received 2008-12-04, accepted 2009-10-15

\begin{abstract}
After more than 40 years of interruption new field observations have been made by an E-54 type torsion balance (TB)in the Csepel-island. These TB measurements were accompanied by a detailed gravimetric survey of each station with LCR gravimeters. Both vertical (VG) and horizontal $(H G)$ gravity gradients were determined at each TB station for $V G$ interpolation and reliability tests.

Vertical gradient of gravity cannot be measured directly by the Eötvös TB. However, we successfully interpolated VG differences in the network of TB measurements following the idea originally due to Haalck. Reliability tests by comparing $H G$ and VG gravimetric and TB measurements were also performed. Our recent paper discusses first results of these TB and gravimetric measurements which are scheduled to be continued in the future as well.
\end{abstract}

\section{Keywords \\ gravity gradients $\cdot$ gravimeter $\cdot$ Eötvös torsion balance}

\section{Acknowledgement}

The authors wish to thank K. Kloska for the contribution to the measurements. This research was funded by the OTKA projects $K 60657$ and K76231, which is hereby gratefully acknowledged.

\section{Géza Csapó}

Geophysical Institute, ELTE, H-1145 Budapest, Kolumbusz u. 17-23., Hungary

\section{Sándor Laky}

Research Group of Physical Geodesy and Geodynamics of the Hungarian Academy of Sciences, POBox91, H-1521, Hungary

\section{Csaba Égető}

Zita Ultman

Department of Geodesy and Surveying, BME, PO Box 91, H-1521,, Hungary

\section{Gyula Tóth}

\section{Lajos Völgyesi}

Department of Geodesy and Surveying, Research Group of Physical Geodesy and Geodynamics of the Hungarian Academy of Sciences, BME, POBox91, H1521, Hungary

\section{Introduction}

The purpose of the research presented in this paper is to use Hungarian torsion balance (TB) measurements in improved gravity field determination for Hungary in addition to a number of (about 300000) gravity data determined by gravimetry. There are about 60000 torsion balance stations in Hungary that have been measured primarily for prospecting of raw materials. Our previous studies e.g. Tóth, 2007 [7] and geodetic processing of torsion balance measurements made by Loránd Eötvös himself showed that these measurements are suitable for gravity field determination. Also, it is possible to generate all functional of the gravity field by combining TB data with gravimetry. In order to verify these theoretical results in practice and to compare the two (gravimetric and gradiometric) gravity field determination techniques several tests have been made.

\section{Determination of the horizontal gradient of gravity}

We measured a test network of $300 \mathrm{~m}$ x $300 \mathrm{~m}$ spacing both by the balance and gravimeters in a flat terrain about $60 \mathrm{~km}$ south from Budapest, at the southern part of the Csepel-island. Distribution of points on the test area can be seen on Fig. 11. The

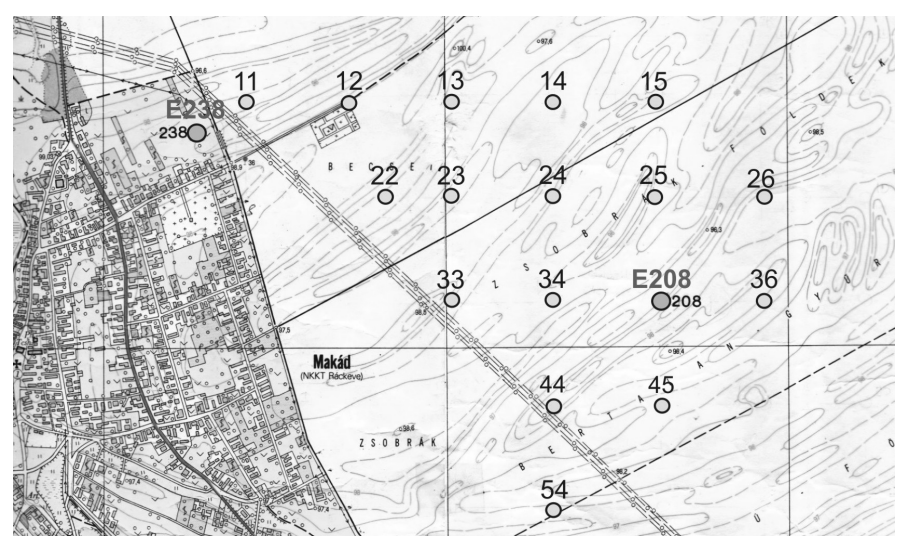

Fig. 1. Location of the torsion balance stations on the test area

horizontal gradients of gravity $\mathrm{HG}$ determined by gravimeters and torsion balance are denoted by $g_{s}$ and $W_{z s}$ respectively:

$$
g_{s}=\frac{\partial g}{\partial u}=\sqrt{\left(\frac{\partial g}{\partial x}\right)^{2}+\left(\frac{\partial g}{\partial y}\right)^{2}}=\sqrt{g_{x}^{2}+g_{y}^{2}},
$$




$$
W_{z s}=\frac{\partial^{2} W}{\partial z \partial u}=\sqrt{\left(\frac{\partial^{2} W}{\partial z \partial x}\right)^{2}+\left(\frac{\partial^{2} W}{\partial z \partial y}\right)^{2}}=\sqrt{W_{z x}^{2}+W_{z y}^{2}},
$$

where $g_{x}, g_{y}$ and $W_{z x}, W_{z y}$ are the $N-S$ and $E-W$ components of the horizontal gradients of gravity (the positive $x$-axis points towards North and positive $y$-axis points towards East) and in an arbitrary direction of $u$ according to the usual notation [9]:

$$
W_{z u}=\frac{\partial^{2} W}{\partial z \partial u}=\frac{\partial g}{\partial u}=g_{u} .
$$

Gravimetric measurements were performed with double star method $(P-N-P-E-P-S-P-W-P-\ldots)$ each of the four compass points $(N, E, S, W)$ were located at $5 \mathrm{~m}$ distance from the centre point $P$ (Fig. 2). $\Delta g$ values between these points were divided by $5 \mathrm{~m}$ to yield components of $H G$ in $\mu \mathrm{Gal} / \mathrm{m}$. Standard errors

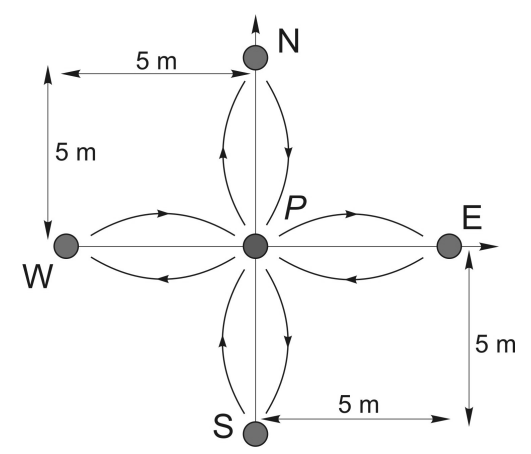

Fig. 2. Gravimetric HG measurements by double star method

of $g_{s}$ were calculated from repeated measurements according to the formula

$$
\sigma_{g_{s}}=\sqrt{\sigma_{g_{x}}^{2}+\sigma_{g_{y}}^{2}}
$$

We used LCR gravimeters equipped with electronic levels and capacitance beam position indicator (CPI) electronics, and readings were taken by interpolation method on a digital multimeter with external R-C filter [3].

The mean standard deviation of our gravimetric measurements by LCR gravimeters for the horizontal gradients of gravity was $\pm 5 \mu \mathrm{Gal}\left(1 \mu \mathrm{Gal}=10^{-8} \mathrm{~m} / \mathrm{s}^{2}\right)$. This is better than the usual accuracy of gravity field measurements. The lower error can be attributed to the fast relocation of the instrument between measurement points with negligible adverse mechanical effects, and also to the fact that only minimal dial turning was necessary before taking readings. Gravity measurements on certain sites after several days with different weather circumstances were repeated (see Table 1 points 22 and 33). Under favourable conditions (point 33) we found a good agreement, whereas for entirely different circumstances (point 22) the discrepancies were higher.

We make a remark, however, for the rating of these results. The external conditions were very unfavorable for the TB measurements. The temperature in the observation hut increased rapidly by $7-8^{\circ} \mathrm{C}$ during the measurement and in certain days reached even $40^{\circ} \mathrm{C}$ whereas the vertical temperature gradient
Tab. 1. Horizontal gravity gradients on the test area $\left(g_{s}\right.$ from measurements of gravimeters, $W_{z s}$ from torsion balance observations in Eötvös Unit*) and

\begin{tabular}{|c|c|c|c|c|c|}
\hline \multirow{2}{*}{ Point } & \multirow{2}{*}{$\begin{array}{l}g_{s} \\
{[E]^{*}}\end{array}$} & \multirow{2}{*}{$\frac{W_{z s}}{[\mathrm{E}]^{*}}$} & \multirow{2}{*}{ diff. $[E]^{*}$} & \multirow{2}{*}{ remarks } & \multirow{2}{*}{$\begin{array}{l}\Delta H_{\max } \\
{[\mathrm{m}]}\end{array}$} \\
\hline & & & & & \\
\hline E238 & $6.5 \pm 8.4$ & $1.7 \pm 0.2$ & 4.8 & & 1.95 \\
\hline E208 & $8.4 \pm 2.4$ & $3.3 \pm 0.6$ & 5.1 & & 2.16 \\
\hline 11 & $6.4 \pm 3.2$ & - & & & 1.83 \\
\hline 12 & $10.6 \pm 5.6$ & $5.2 \pm 0.6$ & 5.4 & & 2.98 \\
\hline 13 & $10.2 \pm 12.6$ & $2.3 \pm 0.2$ & 7.9 & & 1.63 \\
\hline 14 & $1.1 \pm 2.2$ & $0.4 \pm 1.2$ & 0.7 & & 0.61 \\
\hline 15 & $1.5 \pm 2.8$ & $2.8 \pm 0.6$ & -1.3 & & 1.35 \\
\hline 22 & $8.7 \pm 5.4$ & - & & & \\
\hline 22 & $5.8 \pm 5.7$ & $1.6 \pm 0.2$ & 4.2 & repeated & 2.51 \\
\hline 23 & $6.3 \pm 5.7$ & $1.4 \pm 0.6$ & 4.9 & & 1.52 \\
\hline 24 & $4.5 \pm 4.3$ & $0.8 \pm 0.5$ & 3.7 & & 1.49 \\
\hline 25 & $3.8 \pm 8.4$ & $2.7 \pm 0.5$ & 1.1 & & 2.63 \\
\hline 26 & $2.5 \pm 3.6$ & $1.7 \pm 0.5$ & 0.8 & & 2.31 \\
\hline 33 & $4.8 \pm 3.3$ & $1.8 \pm 0.2$ & 3.0 & & \\
\hline 33 & $4.1 \pm 3.2$ & - & & repeated & 1.60 \\
\hline 34 & $0.5 \pm 4.8$ & $2.4 \pm 1.0$ & -1.9 & & 1.24 \\
\hline 36 & $5.0 \pm 3.9$ & $2.2 \pm 0.5$ & 2.8 & & 1.44 \\
\hline 44 & $5.5 \pm 3.6$ & $0.7 \pm 0.3$ & 4.8 & & 1.96 \\
\hline 45 & $4.9 \pm 5.7$ & $1.2 \pm 0.4$ & 3.7 & & 0.52 \\
\hline 54 & $2.4 \pm 5.0$ & $1.3 \pm 0.6$ & 1.1 & & 1.38 \\
\hline \multicolumn{2}{|c|}{ mean std.: $\pm \mathbf{5 . 0}$} & mean diff.: & & & \\
\hline
\end{tabular}
$\Delta H_{\max }$ are the maximum height differences all around the points up to the horizontal distance of $100 \mathrm{~m}$.

was $7-8^{\circ} \mathrm{C} / \mathrm{m}$. The latter figure presumably caused adverse air turbulence inside torsion wire protecting tubes.

Observations by the E-54 type torsion balance in 5 azimuths have been made by taking visual readings and after this repeated readings in the first two azimuths concluded the measuring series. First readings were taken 40-50 minutes after releasing the arrester of torsion wires, and hereinafter the readings were taken in a regular 40-minute pattern. The rotation of the beam was provided by mechanical clockwork. It follows from the construction of the double balance that each output quantity comes from two - partly independent - observations. The final result was the arithmetic mean referring for these two balances. The standard deviations of horizontal gravity gradients determined by the torsion balance were estimated from the differences and the effect of the observer's mass was also investigated (Figs. 3 and 4). This mass effect during a 2-minute observation time was found to be about 0.4-0.6 E on average. These results were obtained by using an automated CCD reading system.

The mean difference between gravimeter and torsion balance measurements is $3 \mu \mathrm{Gal} / \mathrm{m}$ and this figure is smaller than the standard deviation of gravimeter measurements.

The last column of Table 1 contains maximum height differences $\Delta H_{\text {max }}$ of the topographic survey carried out in 8 azimuths around each measurement site up to the maximum distance of $100 \mathrm{~m}$. The maximum $\Delta H$ for the cca $3 \mathrm{~km}^{2}$ test area is 


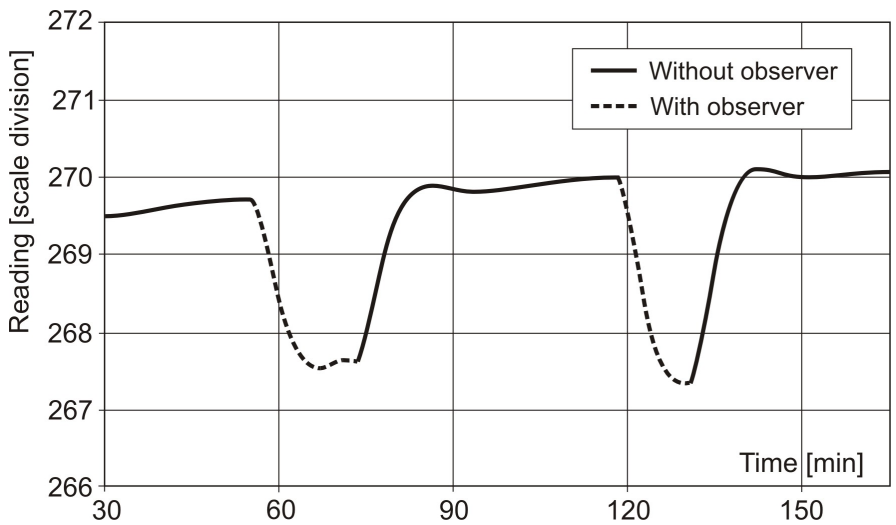

Fig. 3. Effect of observer's mass on the torsion balance readings
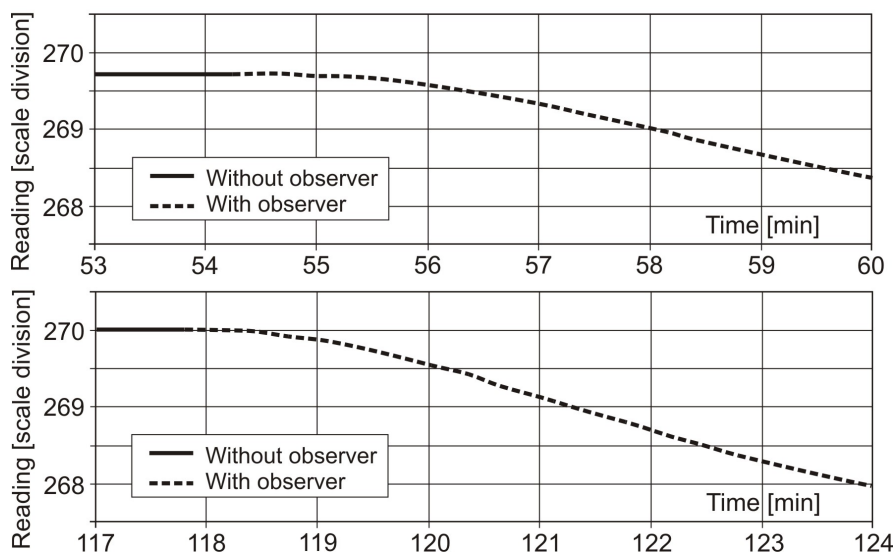

Fig. 4. Magnified parts of the effect of observer's mass on the torsion balance readings

$3.67 \mathrm{~m}$. Effect of the nearby terrain was negligible; the ground was carefully leveled around each site.

From the comparison of TB and gravimetric determination of the horizontal gravity gradient it can be concluded that by LCR gravimeters equipped with electronic levels and CPI electronics and readings taken on a digital multimeter it is impossible to have a reliable determination of HG. It is still best determined by the torsion balance.

\section{Determination of gravity differences $\Delta \boldsymbol{g}$ by gravime- try and the torsion balance}

Gravity differences $\Delta g$ between points were determined by LCR gravimeters mentioned in the previous section. Measurements were performed between 16 points at 4 different epochs and measuring series - simultaneously with 2 LCR gravimeters. These gravimeters were transported by a car between measurement points and base stations of measuring series. (Since all network points were mounted in plough-land, gravimeters were constantly put under adverse mechanical stress - shock by the transportation.)

The measurements were processed with tidal, instrument height, barometric and drift corrections. (Non-modeled effects - thermal and mechanical stress - were reckoned in the drift correction.)

The gravity differences $\Delta g$ shown in Table 2 are raw (nonadjusted) values. It can be recognized from data in the differ- ences (diff.) column that there is no scale bias between the two gravimeters.

Tab. 2. Unadjusted gravity differences of the test network

\begin{tabular}{llrrrr}
\hline \multirow{2}{*}{ connection } & distance & $\begin{array}{r}\Delta g \\
\text { LCR-220 }\end{array}$ & $\begin{array}{c}\Delta g \\
\text { LCR-1919 }\end{array}$ & diff. & mean \\
\cline { 3 - 6 } & {$[\mathrm{m}]$} & \multicolumn{5}{c}{$[\mathrm{mGal}]$} \\
\hline E238-12 & 451.961 & 0.318 & 0.342 & -0.024 & 0.330 \\
$12-11$ & 298.924 & -0.369 & -0.371 & +0.002 & -0.370 \\
$11-13$ & 599.540 & 1.105 & 1.085 & +0.020 & 1.095 \\
$13-14$ & 298.737 & 0.593 & 0.578 & +0.015 & 0.586 \\
$14-15$ & 298.043 & 0.528 & 0.529 & -0.001 & 0.528 \\
$15-E 208$ & 599.259 & 0.636 & 0.649 & -0.013 & 0.642 \\
E238-E208 & 1437.610 & 2.834 & 2.797 & +0.037 & 2.816 \\
$23-24$ & 299.136 & 0.314 & 0.324 & -0.010 & 0.319 \\
$24-25$ & 300.904 & 0.264 & 0.233 & +0.031 & 0.248 \\
$25-26$ & 300.060 & 0.230 & 0.287 & -0.057 & 0.258 \\
$36-34$ & 602.220 & -0.645 & -0.664 & +0.019 & -0.654 \\
$34-33$ & 299.760 & -0.190 & -0.195 & +0.005 & -0.192 \\
$33-22$ & 356.443 & -0.812 & -0.780 & -0.032 & -0.796 \\
$12-23$ & 424.380 & 1.295 & 1.256 & +0.039 & 1.276 \\
$23-33$ & 298.010 & 0.407 & 0.438 & -0.031 & 0.422 \\
$54-44$ & 424.265 & -0.364 & -0.328 & -0.036 & -0.346 \\
\hline
\end{tabular}

Gravity differences $\Delta g$ between stations can be evaluated upon the assumption that distances and height differences are small [10]. In this case horizontal gradients measured at two sites can be replaced by their mean values along the connecting line. Moreover, the effect of vertical gravity gradient on $\Delta g$ was not considered. Hence the following approximate formula for obtaining the gravity difference $\Delta g$ between two points $A$ and $B$ can be written:

$$
\Delta g_{A B}=\frac{\left(W_{z s}\right)_{A}+\left(W_{z s}\right)_{B}}{2} t_{A B} .
$$

Here $\left(W_{z s}\right)_{A}$ and $\left(W_{z s}\right)_{B}$ are the components of gravity gradients along the direction of $A B$ measured at the two points, respectively, while $t_{A B}$ denotes the distance between the two points. The result is expressed in $\mathrm{mGal}\left(1 \mathrm{mGal}=10^{-5} \mathrm{~m} / \mathrm{s}^{2}\right)$.

When gravity differences $\Delta g$ between the test network points were computed as above, it was observed that the variation of horizontal gravity gradients are not linear between the points. Hence, we followed another approach for determining the figures shown in Table 3 . First, a digital terrain model (DTM) with grid spacing of $5 \mathrm{~m} \times 5 \mathrm{~m}$ was constructed from 1:10000 scale topographic maps and a topographic survey carried out with total stations. Second, a mass model and a reference mass model with average height of the area were constructed from triangular prisms with uniform $2000 \mathrm{~kg} / \mathrm{m}^{3}$ density. Third, gravity gradients were determined by forward gravity field modeling using formulas by Holstein, 2003 [5] for a grid with $50 \mathrm{~m} \times 50 \mathrm{~m}$ spacing, and subsequently these gradients were used for interpolation of the nonlinear change of gravity gradients between several network points in Table 3 . Finally, gravity difference $\Delta g$ was computed between two such points as a sum of individual contributions $\Delta g_{i}$. 
Tab. 3. Comparison of $\Delta g$ from gravimetry and from torsion balance with nonlinear correction over the network

\begin{tabular}{|c|c|c|c|}
\hline connection & $\begin{array}{r}\Delta g \text { (gravimeter) } \\
\text { [mGal] }\end{array}$ & $\begin{array}{r}\Delta g(\mathrm{~TB}) \\
\text { [mGall] }\end{array}$ & $\begin{array}{r}\text { difference } \\
\text { [mGal] }\end{array}$ \\
\hline E238-12 & 0.330 & 0.199 & 0.131 \\
\hline $12-11$ & -0.370 & -0.168 & -0.202 \\
\hline $11-13$ & 1.095 & 0.454 & 0.641 \\
\hline $13-14$ & 0.586 & 0.166 & 0.420 \\
\hline $14-15$ & 0.528 & 0.084 & 0.444 \\
\hline 15-E208 & 0.642 & 0.323 & 0.319 \\
\hline E238-E208 & 2.816 & 1.057 & 1.759 \\
\hline $23-24$ & 0.319 & 0.176 & 0.143 \\
\hline $24-25$ & 0.248 & 0.053 & 0.195 \\
\hline $25-26$ & 0.258 & 0.208 & 0.050 \\
\hline $36-34$ & -0.654 & -0.116 & -0.538 \\
\hline $34-33$ & -0.192 & -0.218 & 0.026 \\
\hline 33-22 & -0.796 & -0.313 & -0.483 \\
\hline $12-23$ & 1.276 & 0.457 & 0.819 \\
\hline $23-33$ & 0.422 & 0.158 & 0.264 \\
\hline
\end{tabular}

To asses the accuracy of $\Delta g$ from torsion balance measurements the following factors should be considered:

1-the horizontal position error is $1-2 \mathrm{~cm}$

2-the standard error of torsion balance measurements is 5-6 E

$3-5 \mathrm{~cm}$ distance $\left(t_{A B}\right)$ error generates cca. $1.6 \mu \mathrm{Gal}$ error

$4-5^{\circ}$ azimuth error produces cca. $1.3 \mu \mathrm{Gal}$ error

We processed torsion balance observations at all of the 17 sites without drift and with assuming linear drift of the torsion wires. The accuracy depends on several factors. One of these is the visual readout error. If we estimate it to be about $0.2-0.3$ scale division, it produces 0.8-1.1 E and 1.7-3 E error of gravity gradients $\left(W_{z x}, W_{z y}\right)$ and curvature values $\left(W_{\Delta \mathrm{g}}=W_{y y}-W_{x x}\right.$, $\left.2 W_{x y}\right)$, respectively. The error due to the nonlinear drift of torsion wires is more severe. Readings captured with a video camera showed a nonlinear initial drift of 0.7 scale division, which may readily produce an error figure twice of the above. The accuracy achieved can be estimated from the sample standard deviation of differences between the two balances of the double balance. The following standard deviations and mean differences were computed by processing 7 measurements in 5 azimuths with the linear drift model (Table 4).

Tab. 4. Mean and standard deviation of differences between the two balances at all of the 17 sites $\left(1 E=1\right.$ Eötvös Unit $\left.=10^{-9} s^{-2}\right)$

\begin{tabular}{lll}
\hline & $\begin{array}{l}\text { standard } \\
\text { deviation }[\mathrm{E}]\end{array}$ & mean [E] \\
\hline$W_{z x}$ & \pm 5.3 & 3.8 \\
$W_{z y}$ & \pm 6.2 & -4.1 \\
$W_{\Delta}$ & \pm 4.8 & -1.0 \\
$2 W_{x y}$ & \pm 6.8 & -0.4 \\
\hline
\end{tabular}

Measurements of the two balances are not entirely indepen- dent since the influence of certain environmental factors (e.g. temperature) on the readings is expected to be about the same. Having said that, however, the accuracy of TB measurements can be estimated from the differences: mean standard deviation of differences at 17 stations is $\pm 6 \mathrm{E}( \pm 0.6 \mu \mathrm{Gal} / \mathrm{m})$ with significant variation from one site to the other (extreme: $\pm 1.7 \mathrm{E}$ and $\pm 12.4 \mathrm{E})$. Absolute values of the estimated linear drift also showed major variation of $0.1-1.7$ scale division (s.d.) during the $40 \mathrm{~min}$ readout interval. From inspection of Table 4 it is evident that gradients of the two balances have a significant non-zero mean. Miscalibration or a yet undiscovered factor can be the culprit. A new calibration of the instrument is planned to settle this issue.

Another source of errors is scale misreading. Site 14 may be an example of this, since a linear drift model yielded worse results than the no-drift model, and particularly at this site differences between the two balances of the double balance produced an extremely high standard deviation of $\pm 12.4 \mathrm{E}$ and the highest drift estimates (-1.7 and -0.9 s.d.).

As it has been mentioned, the observer's mass effect is a significant source of error, producing on average 0.4-0.6 E deviation within a 2-min interval.

Several of the above errors are expected to be eliminated or mitigated by automated reading, a more realistic drift modeling, a better thermal insulation of the observing hut and perhaps thorough a suitable choice of the reading sequence.

Table 3 shows that a reasonable agreement was found at a considerable number of sites between measured and interpolated $\Delta g$ if nonlinear variation of horizontal gravity gradients were taken into account. The cause of larger discrepancies of about $0.2 \mathrm{mGal}$ in this table requires further investigation.

\section{VG determinations in a network of torsion balance (TB) measurements}

It is well-known that vertical gradients of gravity (VG) can not be determined directly from observations of the torsion balance.

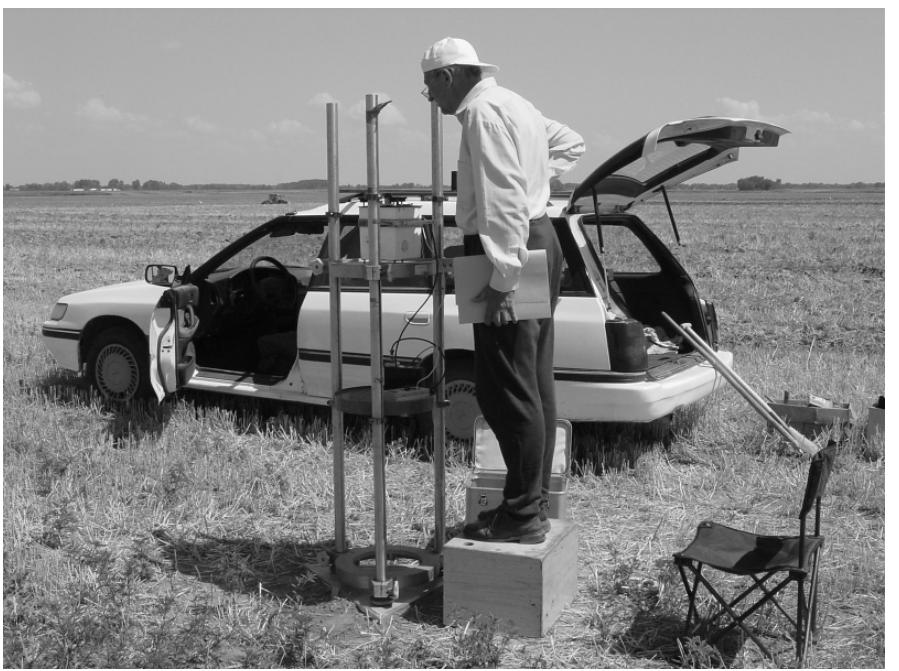

Fig. 5. VG measurement 
Tab. 5. Observed VG data

\begin{tabular}{|c|c|c|c|c|}
\hline \multirow{2}{*}{ site } & \multicolumn{2}{|c|}{ linear approximation } & \multicolumn{2}{|c|}{ quadratic approximation } \\
\hline & VG [mGal/m] & $\operatorname{STD}_{V G}$ & VG [mGal/m] & $\operatorname{STD}_{V G}$ \\
\hline 11 & -0.3078 & $\pm \mathbf{0 . 0 4 1}$ & -0.3120 & 0.041 \\
\hline 12 & -0.3064 & \pm 0.008 & -0.3099 & 0.005 \\
\hline 13 & -0.3084 & \pm 0.011 & -0.3035 & 0.006 \\
\hline 14 & -0.3001 & \pm 0.005 & -0.2982 & 0.003 \\
\hline 15 & -0.3048 & \pm 0.013 & -0.3107 & 0.006 \\
\hline 22 & -0.3053 & \pm 0.008 & -0.3063 & 0.005 \\
\hline 23 & -0.3049 & $\pm \quad 0.009$ & -0.3010 & 0.005 \\
\hline 24 & -0.3066 & \pm 0.006 & -0.3027 & 0.002 \\
\hline 25 & -0.3053 & \pm 0.012 & -0.3109 & \pm 0.006 \\
\hline 26 & -0.3050 & \pm 0.017 & -0.3012 & 0.009 \\
\hline 34 & -0.3030 & \pm 0.012 & -0.3016 & $\pm \quad 0.009$ \\
\hline E208 (35) & -0.3065 & $\pm \quad 0.008$ & -0.3080 & $\pm \quad 0.009$ \\
\hline 44 & -0.3092 & $\pm \quad 0.011$ & -0.3076 & \pm 0.006 \\
\hline 45 & -0.3120 & \pm 0.018 & -0.3190 & $\pm \quad 0.009$ \\
\hline B502 & -0.3189 & $\pm \quad 0.012$ & -0.3202 & \pm 0.007 \\
\hline E238 & -0.3074 & \pm 0.006 & -0.3083 & $\pm \quad 0.007$ \\
\hline mean & -0.3062 & & -0.3067 & \\
\hline
\end{tabular}

There is a certain technique, however, whereby vertical gradients can be calculated at each TB measurement site if one or several VG values are available in the network [7]. To verify this, gravimetric vertical gradients were determined at the majority of TB sites of the test network [2]. Measurements were made by two LCR gravimeters at heights 50, 700 and $1300 \mathrm{~mm}$ above the markers (Fig. 5). Table 5 shows the mean VG determined by the two gravimeters for all the measured sites. Measurement accuracy was strongly degraded by the effect of variable intensity wind-forces (standard deviation (STD) of measurements affected by intense wind-forces are indicated by boldface letters).

The average VG over the test area is close to its normal (nominal) value $(0.3086 \mathrm{mGal} / \mathrm{m})$ and the deviations reach only several $\mu \mathrm{Gal} / \mathrm{m}$ for these sites.

\section{Application of Haalck's method}

An original idea due to Haalck, 1950 [4] enables the determination of missing $W_{z z}$ vertical gravity gradients VG from TB measurements since third derivatives of the geopotential establishes the required link between observed and missing components according to the following formulas:

$$
\begin{aligned}
& W_{z z x}=-W_{\Delta x}-2 W_{x y y} \\
& W_{z z y}=W_{\Delta y}-2 W_{x y x} \\
& W_{z z z}=-W_{x z x}-W_{y z y} .
\end{aligned}
$$

To consider the nonlinear variation of gravity gradients, we prepared grids of gravity gradients with the aid of the digital terrain model of the area with spacing of $50 \mathrm{~m} \times 50 \mathrm{~m}$. By numerical differentiation of these grids the necessary derivatives on the right hand side of (1) were computed, and at a constant height $z$ above the mean sea level - by numerical integration of $W_{z z x}, W_{z z y}$ using formulas of Vassiliou (1986) [8] - we obtained $W_{z z}$. The map of the computed $W_{z z}$ (in E units) can be seen in
Fig. 6, together with measurement sites and topographic survey points. The correlation of computed vertical gradients VG with the DTM of the area (Fig. 7) is remarkable. Several conclusions can be drawn by testing the numerical solution procedure.

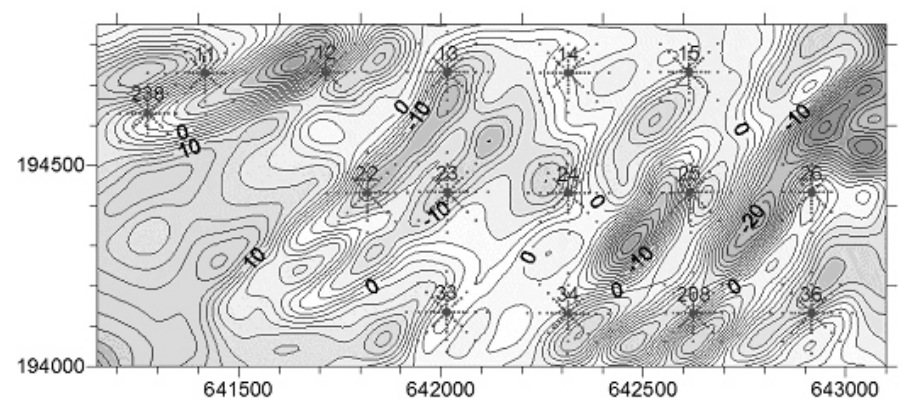

Fig. 6. Interpolated vertical gradients $V G$ for the test area from $T B$ measurements and DTM. Units are $\mathrm{E}\left(1 \mathrm{E}=1 \mathrm{Eötvös}=10^{-9} \mathrm{~s}^{-2}\right)$. Dots are topographic survey points for TB sites

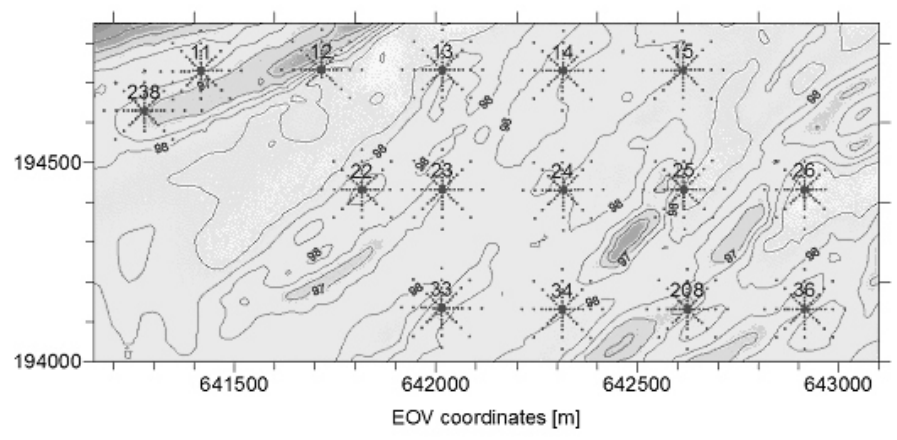

Fig. 7. DTM of the test area. Heights are in [m]. Dots are topographic survey points for TB sites

Tab. 6. Observed VG by quadratic approximation, interpolated VG by Haalck's method and their differences

\begin{tabular}{cccc}
\hline \multirow{2}{*}{ site } & observed VG & interpolated VG & differences \\
\cline { 2 - 4 } & \multicolumn{3}{c}{$[\mathrm{mGal} / \mathrm{m}]$} \\
\hline 11 & -0.3120 & -0.3120 & 0.0000 \\
12 & -0.3099 & -0.3122 & -0.0023 \\
13 & -0.3035 & -0.3113 & -0.0078 \\
14 & -0.2982 & -0.3116 & -0.0134 \\
15 & -0.3107 & -0.3130 & -0.0023 \\
22 & -0.3063 & -0.3118 & -0.0055 \\
23 & -0.3010 & -0.3116 & -0.0106 \\
24 & -0.3027 & -0.3111 & -0.0084 \\
25 & -0.3109 & -0.3111 & -0.0002 \\
26 & -0.3012 & -0.3111 & -0.0099 \\
34 & -0.3016 & -0.3103 & -0.0087 \\
E208 (35) & -0.3080 & -0.3114 & -0.0034 \\
mean: & -0.3055 & -0.3115 & STD: -0.006 \\
\hline
\end{tabular}

From Table 6 it appears that interpolated vertical gradients VG are considerably smaller (max./min. $+27 / 28 \mathrm{E}$ for the whole area) than those obtained by gravimeters (cf. Table 5). This fact can be attributed to the applied numerical differentiation scheme (forming central differences), the transfer function of which cuts down the signal at higher frequencies [1], or from the numerical 
integration procedure. The non-linear variation of vertical gradients should also be a factor. The reconstruction of a function from given gradient fields is a well-known problem e.g. in the field of image processing and it leads to the numerical solution of the 2D Poisson equation e.g. [6]. Hence we plan to investigate several other reconstruction techniques to calculate of $W_{z z}$ from its known horizontal gradients.

\section{Conclusions}

Earlier theoretical investigations and geodetic torsion balance measurements made by Lorand Eötvös showed that these measurements are good for gravity field determination as well and it is possible by combining torsion balance with gravimetry to generate all functionals of the gravity field.

From the comparison of torsion balance and gravimetric determination of the horizontal gradients of gravity it can be concluded that it is impossible to have a reliable determination by LCR gravimeters, it is still best determined by torsion balance.

Computing $\Delta g$ from torsion balance measurements we realized, the variations of horizontal gradients of gravity between two points would not be supposed linear neither below of a few hundred meters distance. However, generally better agreement can be seen between the measured and the computed $\Delta g$ taking into account a digital terrain model for estimating the nonlinear variation of the horizontal gravity gradients - but further investigations are necessary to study the reason of the bigger discrepancies.

Comparing the vertical gradients measured by gravimeters and computing from torsion balance measurements various differences can be seen. The reason may come from the applied numerical differentiation scheme, or from the integration procedure.

\section{References}

1 Christmas W J, Spatial filtering requirements for gradient-based optical flow measurement, IEEE Trans. Image Processing 9 (2000), no. 10, 18171820.

2 Csapó G, Effect of vertical gravity gradient on the accuracy of gravimeter measurements based on Hungarian data, Geophysical Transactions 42 (1999), no. 1-2, 67-81.

3 Csapó G, Accuracy Tests of LCR Model G gravimeters, Geophysical Transactions 45 (2006), no. 3, 123-134.

4 Haalck H, Die vollständige Berechnung örtlicher gravimetrischer Störefelder aus Drehwaagemessungen, 4, Veröffentlichungen des Geodätischen Institutes Potsdam, 1950.

5 Holstein $\mathbf{H}$, Gravimagnetic anomaly formulas for polyhedra of spatially linear media, Geophysics 68 (2003), 157-167, DOI 10.1190/1.1543203.

6 Simchony T, Chellappa R, Shao M, Direct analytical methods for solving Poisson equations in computer vision problems, IEEE Trans. Pattern Anal. Machine Intell. 12 (1990), 435-446, DOI 10.1109/34.55103.

7 Tóth Gy., Vertical gravity gradient determination in a network of torsion balance measuring sites, Geomatikai Közlemények X (2007), 29-36.

8 Vassiliou A. A, Numerical techniques for processing airborne gradiometer data, 4, UCSE Report 20017, Dept. of Surv. Eng., University of Calgary, Calgary, Alberta, 1986.

9 Völgyesi L., Geophysics, Múegyetemi kiadó, 2002.

10 Völgyesi L., Tóth Gy., Determination of gravity anomalies from torsion balance measurements., Gravity, Geoid and Space Missions GGSM 2004., 2004, pp. 368. 